\title{
Evidence for the black hole event horizon
}

\section{Citation}

Narayan, Ramesh. 2003. "Evidence for the Black Hole Event Horizon." Astronomy and

Geophysics 44 (6): 6.22-6.26. https://doi.org/10.1046/j.1468-4004.2003.44622.x.

\section{Permanent link}

http://nrs.harvard.edu/urn-3:HUL.InstRepos:41384887

\section{Terms of Use}

This article was downloaded from Harvard University's DASH repository, and is made available under the terms and conditions applicable to Other Posted Material, as set forth at http:// nrs.harvard.edu/urn-3:HUL.InstRepos:dash.current.terms-of-use\#LAA

\section{Share Your Story}

The Harvard community has made this article openly available.

Please share how this access benefits you. Submit a story.

Accessibility 


\title{
Evidence for the Black Hole Event Horizon ${ }^{1}$
}

\author{
Ramesh Narayan \\ Department of Astronomy, Harvard University, Harvard-Smithsonian Center for \\ Astrophysics, 60 Garden Street, Cambridge, MA 02138, U.S.A.
}

\begin{abstract}
Astronomers have discovered many candidate black holes in X-ray binaries and in the nuclei of galaxies. The candidate objects are too massive to be neutron stars, and for this reason they are considered to be black holes. While the evidence based on mass is certainly strong, there is no proof yet that any of the objects possesses the defining characteristic of a black hole, namely an event horizon. Type I X-ray bursts, which are the result of thermonuclear explosions when gas accretes onto the surface of a compact star, may provide important evidence in this regard. Type I bursts are commonly observed in accreting neutron stars, which have surfaces, but have never been seen in accreting black hole candidates. It is argued that the lack of bursts in black hole candidates is compelling evidence that these objects do not have surfaces. The objects must therefore possess event horizons.
\end{abstract}

\section{Introduction}

The story of astrophysical black holes begins with a simple question: How does a star, or any other gravitating object, hold itself up against its own self-gravity? In the case of the Sun, the answer is easy. The Sun is hot because of thermonuclear reactions in its interior, and the resulting thermal pressure counteracts the compressive action of gravity. The same is true of all the stars we see shining in the night sky. When a star runs out of nuclear fuel and dies, it must find other ways to fight gravity. Dead stars with masses up to the Chandrasekhar limit, $M_{\mathrm{Ch}}=1.4 M_{\odot}$, become white dwarfs, where electron degeneracy supplies the necessary pressure. Above the Chandrasekhar limit, and up to a second mass limit, $M_{\mathrm{NS} \text {, max }} \sim 2-3 M_{\odot}$, dead stars become neutron stars, where neutron degeneracy pressure holds them up. But that, according to conventional physics, is the end of the road.

\footnotetext{
${ }^{1}$ George Darwin Lecture presented at the Royal Astronomical Society, London, December 13, 2002.
} 
If a dead star has a mass $M>M_{\mathrm{NS} \text {,max }}$, there is no known force that can hold the star up. What we have then is a black hole, one of the most extraordinary concepts in physics.

A black hole represents the ultimate victory of gravity, where all the mass in the object collapses down to a "singularity", a true geometrical point (at least within classical physics). The object has no material surface. Instead, surrounding the singularity is an "event horizon", which plays the role of a virtual surface. The event horizon is a one-way membrane through which matter and energy can fall in from the outside, but nothing, not even light, can escape from within. The region inside the horizon is thus causally cut off from the outside world. In a real sense, the horizon serves as an effective surface, even though there is no actual material there. For a non-spinning black hole of mass $M$, the radius of the horizon is given by Schwarzschild's result, $R_{S}=2.95\left(M / M_{\odot}\right) \mathrm{km}$. Spinning black holes have a somewhat smaller radius for the same mass.

Since Nature almost certainly makes dead stars with $M>M_{\mathrm{NS} \text {,max }}$, black holes must exist in our Galaxy and in other galaxies. Finding these black holes has been a central goal of high energy astrophysicists for the last few decades, and indeed dozens of excellent candidates have been discovered, many in the last ten years or so. These discoveries are briefly summarized in $\S 2$. We then discuss in $\S \S 3-5$ the main theme of this Lecture: How can we confirm that the black hole candidates discovered by astrophysics are truly black holes? Specifically, do the objects possess the defining characteristic of a black hole, namely an event horizon?

\section{Astrophysical Black Holes}

Many excellent black hole candidates have been discovered in a class of objects called X-ray binaries. These are double stars in which a compact primary star, either a neutron star or a black hole, accretes mass from a normal secondary companion star (see Fig. 1), and radiates the accretion luminosity in X-ray, ultraviolet and optical radiation.

A particular class of X-ray binaries, called soft X-ray transients or SXTs for short, has turned out to be especially helpful in the hunt for black holes. In these binaries, the mass accretion rate $\dot{M}$, and consequently the accretion luminosity $L_{\text {acc }}$, vary with time. For most of the time, an SXT is in a very low luminosity state with $L_{\text {acc }} \sim 10^{-6}-10^{-8} L_{\text {Edd }}$, where $L_{\text {Edd }}=1.25 \times 10^{38}\left(M / M_{\odot}\right) \operatorname{erg~s}^{-1}$ is the Eddington luminosity and $M$ is the mass of the accreting star. Every once in a while, however, the system goes into an accretion outburst and becomes very bright, with a luminosity almost equal to $L_{\text {Edd }}$. It is during the accretion outburst that the binary is usually discovered. After the outburst, the luminosity slowly 
declines over a period of several months, and the binary reverts to quiescence until the next accretion outburst.

Whereas the optical light from an SXT during outburst comes mostly from the accretion disk, the emission during quiescence is dominated by the secondary star. Therefore, in quiescence, it is possible to observe the absorption lines in the stellar spectrum and use the Doppler shifts on these to measure the orbital motion of the secondary. From the orbital period $P_{\text {orb }}$ of the binary and the semi-amplitude $K_{s}$ of the secondary's line-of-sight velocity, one can then obtain the mass function

$$
f(M) \equiv \frac{P_{\mathrm{orb}} K_{s}^{3}}{2 \pi G}=\frac{\sin ^{3} i}{\left(1+M_{s} / M_{p}\right)^{2}} M_{p}
$$

where $M_{s}$ and $M_{p}$ are the masses of the secondary and the compact primary, respectively, $i$ is the inclination angle of the binary orbit, and the final relation on the right is derived by applying Newton's laws of motion to a binary system bound by gravity in a circular orbit. Regardless of the values of $M_{s}$ and $i$, one sees that $f(M)$ is a strict lower limit on $M_{p}$.

McClintock \& Remillard (1986) showed that the SXT A0620-00 has a mass function close to $3 M_{\odot}$, above the likely maximum mass $M_{\mathrm{NS} \text {,max }}$ of a neutron star. A0620-00 was the first truly bona fide black hole candidate, and the sensational discovery of this object opened up a new avenue in the hunt for black holes. In the years since, a dozen or more additional black hole candidates have been discovered in SXTs, all identified as having mass functions $f(M) \gtrsim M_{\mathrm{NS}, \max }$. Special mention should be made of the SXT V404 Cyg (Casares, Charles \& Naylor 1992) which for many years was the champion among black hole candidates, with the largest known mass function of $6.1 M_{\odot}$. Clearly, the compact stars in A0620-00, V404 Cyg, and the other SXTs with large mass functions, are all excellent black hole candidates. In a few SXTs and other kinds of X-ray binaries, $f(M)$ itself is not very large, but there are independent estimates of $i$ and $M_{s}$ which, combined with the measured $f(M)$, indicate that $M_{p}>M_{\mathrm{NS}, \max }$. These objects are also very good black hole candidates, though clearly not as good as the systems with large mass functions. Narayan, Garcia \& McClintock (2002) list the best black hole SXTs as of 2001.

The typical black hole mass in SXTs is $\sim 5-15 M_{\odot}$. In addition to these stellarmass black holes, supermassive black holes have been identified in the nuclei of galaxies. At the center of our own Milky Way Galaxy, for instance, the object Sagittarius A* has been very convincingly shown to be dark and compact, and to have a mass $\sim 4 \times 10^{6} M_{\odot}$ (see the remarkable observations described in Schödel et al. 2002 and Ghez et al. 2003). Another excellent candidate is in the nucleus of the galaxy NGC 4258 (Miyoshi et al. 1995). A couple of dozen additional candidates have been confimed in the nuclei of other nearby galaxies (Gebhardt et al. 2003, and references therein), and today it is generally agreed that 
virtually every galaxy in the universe has a supermassive dark compact mass in its nucleus.

\section{Is This Enough?}

As the above summary indicates, astrophysicists have certainly discovered many compact stars that are too massive to be neutron stars. Can we therefore claim victory in the search for black holes? In the opinion of the speaker, it would be premature to do so.

It is true that physics, specifically General Relativity combined with our knowledge of the properties of matter up to nuclear density, tells us that a compact star with $M>M_{\mathrm{NS}, \max }$ cannot be a neutron star and must therefore be a black hole. However, what makes a black hole unique - indeed the reason why astronomers spend enormous resources searching for it, and the public is so fascinated by it - is not that it is "not a neutron star" but that it possesses an event horizon. It is the event horizon that makes the black hole so special. Therefore, before claiming victory, it would be prudent to look for independent evidence that the black hole candidates identified so far on the basis of their masses actually do have event horizons. The speaker and his collaborators, Dr. Michael Garcia, Dr. Jeremy Heyl and Dr. Jeffrey McClintock, have searched for such evidence during the last several years.

What would constitute plausible evidence? In brief, we need an observed phenomenon - or lack of it - that is a unique signature of an event horizon. Ideally, we should compare black hole candidates to a control sample of objects, say neutron stars, that are known to have surfaces. We should show that some observable characteristic is distinctly different in the two classes of objects, and that the difference is consistent with the notion that one class (black hole candidates) has event horizons and the other class (neutron stars) has surfaces. Furthermore, the difference should not have any other plausible explanation. Of course, for this line of argument to work, we need a working definition of what is plausible and what is not. This tends to be somewhat subjective, though scientists by and large agree on the matter.

X-ray binaries are particularly good for such investigations since some X-ray binaries contain black hole candidates and some contain neutron stars. It is thus possible to find two well-matched sets of binaries, one with black hole candidates that we think may have event horizons, and the other with neutron stars that we know for sure have surfaces. The speaker and his collaborators pioneered such comparisons of matched black hole and neutron star X-ray binareis, and showed in an early study that quiescent black hole SXTs are very much dimmer than quiescent neutron star SXTs (Narayan, Garcia \& McClintock 1997). The latest observations are summarized in Fig. 2, taken from McClintock et al. (2003). As explained 
in Garcia et al. (2001) and Narayan et al. (2002), the large luminosity difference - a factor of 100 to 1000 in Eddington units - is natural if black hole candidates have event horizons. Various alternative explanations have been advanced in an effort to get around this argument, but many of these explanations appear to be ruled out by subsequent observations (Narayan et al. 2002).

Following our initial work, other signatures have been identified that distinguish black hole and neutron star binaries. Sunyaev \& Revnivtsev (2000) showed that neutron star systems have strong variability up to frequencies $\sim 1 \mathrm{kHz}$, presumably from boundary layer radiation close to the stellar surface, whereas black hole systems have a significant decline in flux variations above $\sim 10-50 \mathrm{~Hz}$, presumably because they have no surface and hence do not possess boundary layers. In another study, Done \& Gierlinsky (2003) considered the luminosities and spectra of black hole and neutron star binaries and showed that there are large differences between the two classes. They suggested that the differences arise because black hole candidates have event horizons while neutron stars do not.

We have recently begun a detailed study of Type I X-ray bursts in X-ray binaries with a view to using the bursts to verify the presence of event horizons in black hole candidates. This is the topic of the rest of the Lecture.

\section{Type I X-ray Bursts}

When gas accretes on the surface of a neutron star, it is compressed by the strong surface gravity of the star. As the gas sinks under the weight of continued accretion, it becomes denser and hotter, until the conditions are right for igniting thermonuclear reactions. If the gas consists largely of hydrogen and helium as it usually does, the nuclear burning tends to be unstable (Hansen \& van Horn 1975, see Lewin, Taam \& van Paradijs 1993, Bildsten 1998, for reviews). The instability causes the accreted layer of gas to burn its nuclear fuel explosively within a very short time, leading to a burst of X-ray emission. After the fuel is consumed, the star reverts to its accretion phase, in which fuel accumulates on the surface, until the next thermonuclear instability is triggered. The star thus undergoes a semi-regular series of thermonuclear explosions.

Bursts of X-ray emission from X-ray binaries were first discovered by Grindlay et al. (1976) and were immediately identified with thermonuclear explosions on the surface of a neutron star. These explosions are known as Type I bursts (to distinguish them from a different kind of burst, called Type II bursts, which is not thermonuclear in origin). In a typical Type I burst, the luminosity of the neutron star increases to nearly the Eddington 
limit within less than a second, and the flux then declines over a period of seconds to tens of seconds. The time interval between bursts is usually several hours to perhaps a day or two. Note that Type I bursts are very distinct from the accretion outbursts described earlier. The latter are associated with changes in mass accretion rate and have durations of months, not seconds, and recurrence times of many years, not hours or days.

Type I bursts are very common and have been seen in many neutron star X-ray binaries. However, it is a remarkable fact that no black hole candidate in any X-ray binary has ever had a Type I burst. In some sense, it is obvious that these objects should not experience bursts. A Type I burst requires a surface where matter is compressed and heated until a thermonuclear instability is triggered. A black hole has no surface; matter simply falls in through the event horizon and disappears. Therefore, a black hole cannot have Type I bursts. Since black hole candidates are indeed observed not to have bursts, does it then prove that they have event horizons? The answer is, unfortunately, "No!"

An object that has a Type I burst must have a surface and therefore cannot be a black hole. This statement is uncontroversial. However, an object that does not have Type I bursts does not necessarily lack a surface and therefore is not necessarily a black hole. For instance, there are bona fide neutron star systems, such as most X-ray pulsars, that do not exhibit bursts even though they certainly have surfaces. In order to use the lack of bursts as evidence for the presence of event horizons, we need to carry out the following steps:

(i) Develop a detailed theory of thermonuclear stability of accreting gas on a compact star.

(ii) Use the theory to explain observations of Type I bursts in neutron star systems and, in particular, explain why some neutron stars have bursts and some do not.

(iii) Apply the theory to black hole candidates and demonstrate that these systems would certainly exhibit bursts if they had surfaces.

(iv) Show that the only plausible explanation for the lack of bursts in black hole candidates is that the objects have event horizons, and that all other explanations either are ruled out or are very implausible.

Until we successfully accomplish these four stages, we cannot claim that the lack of bursts in black hole binaries is strong evidence for the event horizon. Over the last two years, we have embarked on a systematic attack on this problem. As part of this work, we have developed a theoretical framework for understanding the stability of nuclear burning on compact stars (Narayan \& Heyl 2002, 2003). 
We follow a star as it accretes gas of specified nuclear composition (typically solar, corresponding to $70 \%$ by mass of hydrogen, $28 \%$ by mass of helium, and $2 \%$ by mass of heavier elements). As the accretion column grows, at each instant we solve for the quasi-equilibrium configuration of the gas layer and obtain the density, temperature and composition of the gas as a function of depth. We then carry out a linear stability analysis to determine if the layer is stable to small perturbations. If for some particular column depth the layer is unstable, we say that the system undergoes a Type I burst. From the amount of unburnt fuel available in the layer at the moment of instability, we estimate the net energy emitted in the burst, and from the column density and the mass accretion rate, we estimate the recurrence time between the bursts. If the accreted layer is stable for all column depths up to some very large value (say $10^{13} \mathrm{~g} \mathrm{~cm}^{-2}$ ), then we say that the system is able to burn nuclear fuel stably and does not undergo bursts. The main innovation in our work is the application of a formal linear stability analysis. The approach is more rigorous than previous analyses, which employed heuristic prescriptions to determine the stability of the accreted gas.

\subsection{Application to Neutron Star X-ray Binaries}

Figure 3 summarizes the results obtained with this model for a neutron star of mass $1.4 M_{\odot}$ and various radii from $10^{0.2} R_{S}=6.5 \mathrm{~km}$ to $10^{0.6} R_{S}=16.4 \mathrm{~km}$. The calculations correspond to a core temperature of $T_{\text {core }}=10^{8} \mathrm{~K}$, which is approximately the temperature expected for a core that cools via the modified URCA process of neutrino emission. This temperature is also close to the upper limit to $T_{\text {core }}$ in neutron star SXTs (see Narayan \& Heyl 2002, 2003). Figure 3 shows that, almost independent of radius, accreting neutron stars are unstable to bursts at low accretion rates $L_{\text {acc }} \lesssim 0.25 L_{\text {Edd }}$ and stable at higher accretion rates. Observations indicate that bursts occur up to a luminosity of about $0.25-0.3 L_{\text {Edd }}$ (van Paradijs et al. 1988; Cornelisse et al. 2003; Tournear et al. 2003), and that systems brighter than this limit have a much reduced bursting frequency. The model is thus consistent with the observations, with a tendency perhaps to underestimate slightly the prevalence of burst behavior.

X-ray pulsars are generally found not to burst. Although these systems are usually not very luminous, nevertheless, because the accretion is channeled onto the magnetic poles by a strong magnetic field, the local mass accretion rate $\dot{\Sigma}\left(\mathrm{g} \mathrm{cm}^{-2} \mathrm{~s}^{-1}\right)$ is generally above the Eddington rate. Therefore, the lack of bursts is consistent with the predictions of the model. In the case of millisecond X-ray pulsars, however, the field is weak and the accretion flow is only mildly channeled. We would expect these sources to burst, and indeed they do.

One could compare model predictions with more detailed observations of burst recur- 
rence times, burst durations, and other observables, as discussed in Narayan \& Heyl (2003). We do not describe the results here, but merely note that the agreement overall is fairly good. In particular, some puzzling observations for bright systems with $L_{\text {acc }}>0.1 L_{\text {Edd }}$, that previously had not been explained, appear to be predicted satisfactorily by the new model. For faint sources with luminosities below about $0.03 L_{\text {Edd }}$ there appear to be some discrepancies in the detailed model predictions, and for $L_{\text {acc }}<0.01 L_{\text {Edd }}$ there are very few observations presently available to check predictions. This could be improved in the future. In any case, we believe we have successfully accomplished stages (i) and (ii) as listed in $\S 4$, and we feel that the model is sufficiently reliable to embark on stage (iii).

\subsection{Application to Black Hole X-ray Binaries}

Let us assume that black hole candidates in SXTs are not black holes, but are some unusual kind of compact objects with surfaces. Would these objects have Type I X-ray bursts? We can answer this question using our burst model.

First, we need to choose the core temperature and the stellar radius. From the very low luminosities of black hole SXTs in quiescence, we know that their core temperatures are no larger than $10^{7.5} \mathrm{~K}$ (Narayan \& Heyl 2002). We present results corresponding to this core temperature, noting that the conclusions do not change very much for lower temperatures. We consider a range of likely radii for the compact mass, from $(9 / 8) R_{S}$ (the smallest allowed radius, see Shapiro \& Teukolsky 1983) up to about $2.8 R_{S}$.

Figure 4 shows the results for a $10 M_{\odot}$ object with a hypothetical surface. We see that such an object would have bursts over a wide range of mass accretion rate. That is, if SXT black hole candidates have surfaces, they ought to produce bursts. Consequently, the absence of bursts is inconsistent with the presence of a surface. This takes care of stage (iii) in $\S 4$ and goes a long way towards proving that black hole candidates must have event horizons. What is left to accomplish is the difficult stage (iv).

\section{Why Do Black Hole Candidates Not Have Type I Bursts?}

The lack of bursts in black hole candidates is certainly consistent with the presence of event horizons in these objects. This is not in dispute. But, is the presence of event horizons the only plausible reason for the lack of bursts? This is the critical question, and it is where the argument becomes most difficult and subjective. The following discussion covers all the possible explanations for the lack of bursts that the speaker considers plausible. 


\subsection{Obvious Explanations}

Could black hole candidates be accreting the wrong kind of nuclear fuel, e.g., something other than hydrogen and helium? This is ruled out because the companion stars that supply the gas are very normal, with standard nuclear abundances. Moreover, a strong hydrogen line is seen in the optical spectra of all black hole SXT accretion disks, confirming the presence of hydrogen in the accreting gas, and helium lines are seen as well in many systems (Dr. J. E. McClintock, private communication).

Could the core temperature be such that bursts are quenched? Our model calculations indicate that bursts are quenched only if the core temperature is above several times $10^{8}$ K (Narayan \& Heyl 2002, 2003). The very low X-ray luminosities of quiescent black hole

SXTs indicate that their cores are much cooler, $T_{\text {core }} \lesssim 10^{7.5} \mathrm{~K}$. So this is not a promising explanation.

Could the burst recurrence times be extremely long, thereby making it very difficult to detect bursts? Figure 5 shows the dependence of the recurrence time on the mass accretion rate for a typical black hole candidate with a radius equal to $2 R_{S}$. The recurrence times are a factor of a few longer than for neutron stars, as one might expect since the accreting objects have larger masses, but the bursts are correspondingly also more powerful by a factor of a few. There is no reason why these bursts should be missed by observations. Figure 5 indicates that the bursts are most easily observed for $L_{\text {acc }} \gtrsim 0.1 L_{\text {Edd }}$. For lower accretion rates, the systems are still burst-unstable (see Fig. 4), but the recurrence times are very long and it is possible to miss these bursts.

\subsection{Wrong Accretion Rate?}

Figures 4 and 5 show that for certain accretion rates very close to the Eddington rate bursts are either absent altogether or are very rare. Could it be that our black hole candidate systems spend all their time in these particular regions of parameter space and that this is why they do not burst? In other words, is it just plain bad luck that we have not seen bursts from these objects? This explanation can be eliminated easily.

Recall from $\S 2$ that all our black hole candidates are found in transient binary systems, so that each of them has experienced one or more accretion outbursts during which its mass accretion rate varied over a wide range. At the start of an accretion outburst, a typical system has a very low accretion rate, off beyond the left edge of Fig. 4. Then, as the outburst builds up, the accretion rate increases and the system moves to the right almost to the right edge of the figure, near the limit of Eddington accretion. This journey typically 
takes about a week. Then, the accretion rate slowly declines over a period of six months or so, during which the system moves slowly back towards the left edge of the figure. Finally, the system plunges back into quiescence, beyond the left edge of the figure.

Figure 5 indicates that there is a favorable range of mass accretion rate, between about 0.1 and 0.6 Eddington, for which bursts are expected to occur quite frequently. Although the particular calculation shown corresponds to a specific choice of the radius, this favorable region of bursting activity is present for all radii that we have tried. Furthermore, most black hole candidates have crossed this region during their accretion outbursts, and each system has spent about a month or so in the region. During this time it should have had several Type I bursts according to the calculations. Therefore, the fact that not a single source has experienced any Type I bursts is very significant, and cannot be "just plain bad luck."

\subsection{Rotation and Magnetic Fields}

Could rapid rotation somehow eliminate bursts? Rotation has the effect of introducing a variation in the effective surface gravitational acceleration $g$ as a function of latitude. However, even a maximally rotating compact star has only a factor of $\sim 2$ variation in $g$ between the equator and the pole. Such a modest variation does not have a serious effect on the burst instability.

Rotation might influence the propagation of the burning front once the instability has been triggered at a single point, and might thereby have an effect on the development of the burst. This is a complex problem since the propagation of deflagration fronts is poorly understood. Although current calculations (e.g., Spitkowsky, Levin \& Ushomirsky 2002), when applied to black hole candidates, seem to indicate that bursts ought to develop readily in these systems, it must still be considered an open question.

Neutron stars with strong magnetic fields do not burst. The accretion flows in these systems are channeled by the field, thereby increasing the effective local mass accretion rate

$\dot{\Sigma}$ to above the Eddington rate. Could black hole candidates have similarly strong fields and thereby avoid bursts? The possibility can be ruled out immediately. When accretion is channeled, the radiation comes out in beams from the magnetic poles, causing the observed $\mathrm{X}$-ray flux to be modulated at the rotation period of the star. This is, in fact, the explanation for the modulations seen in X-ray pulsars. If black hole candidates have strong magnetic fields, they ought to exhibit X-ray pulsations. Since none of these objects has ever shown coherent pulsations, we can rule out the magnetically channeled accretion scenario. In principle, if the magnetic axis is aligned perfectly with the rotation axis there would be 
no observable pulsation, but it requires such an unusual conspiracy in so many objects that we consider it implausible.

\subsection{Exotic Stars}

Conventional physics tells us that black hole candidates cannot be normal compact stars such as white dwarfs or neutron stars. It is therefore reasonable to suppose that, if they are not black holes, then they must be exotic stars of some kind. Could they be exotic in such a manner as to prevent Type I bursts when they accrete gas?

The nature of matter deep in the core of a neutron star is uncertain (Shapiro \& Teukolsky 1983; Glendenning 2000). It might be normal baryonic matter (mostly neutrons plus some protons and electrons), or it might be a pion condensate, or it might be something more unusual like Q-matter (Bahcall, Lynn \& Selipsky 1990), quark matter (Glendenning 2000) or a color-locked superconducting state (Alford, Rajagopal \& Wilczek 1998; Rapp et al. 1998). Could black hole candidates have cores made of some very exotic material, and could this prevent bursts? No, because bursts are very much a surface phenomenon, restricted to low densities $\sim 10^{6} \mathrm{~g} \mathrm{~cm}^{-3}$. The unusual kinds of matter that are invoked for neutron star interiors typically occur at very high pressure, when the density is above nuclear density $\left(\gtrsim 10^{15} \mathrm{~g} \mathrm{~cm}^{-3}\right)$ and certainly above the neutron drip point $\left(\sim 10^{11} \mathrm{~g} \mathrm{~cm}^{-3}\right.$, despite the claim to the contrary by Abramowicz, Kluzniak \& Lasota 2002 who seem to state that the color-locked superconducting state can exist even at low densities). Changes at such high densities and such large depths have no effect on bursts at the surface.

Could black hole candidates be strange stars or some other kind of exotic stars where exotic matter extends all the way to zero pressure at the surface? Models of such stars have indeed been proposed (Bodmer 1971; Witten 1984; see Glendenning 2000). However, when the stars accrete, it is believed that the newly added gas is supported electrostatically as a separate surface layer of normal matter, getting converted to exotic matter only at high density. The low-density layer would behave exactly as in our models and would produce bursts just as in the calculations.

Is there any form of exotic matter that instantly converts infalling baryonic matter into exotic matter even at densities below $10^{6} \mathrm{~g} \mathrm{~cm}^{-3}$ ? If a star were to be made of such material, there would be no bursts since there would be no nuclei on the surface to undergo thermonuclear burning. To the knowledge of the speaker, no such matter is presently known.

Finally, the black hole candidate may consist of some kind of dark matter that does not interact with baryonic gas other than via gravity (just like the dark matter in the universe). 
We could then have a $10 M_{\odot}$ compact object that is completely "porous" to ordinary matter. Infalling gas would fall freely through the dark matter and collect at the center. (The speaker thanks Professor M. J. Rees for suggesting this possibility.) Would such an object have Type I bursts? To answer this question, we need to calculate the radius, surface gravity and redshift of the baryonic gas ball that forms at the center and then check whether or not the object would have Type I bursts. We have carried out some preliminary calculations which suggest that the central gas sphere has characteristics not very different from a neutron star and that the accreting gas does undergo thermonuclear instability and Type I bursts.

\section{Conclusion}

The lack of Type I bursts in black hole SXTs is an important clue to the nature of these compact stars. Based on detailed models we conclude that, if these objects possess surfaces, they should exhibit widespread burst activity. Why then do we not see Type I bursts in black hole SXTs?

We have considered in $\S 5$ a variety of explanations for the lack of bursts and find that most can either be ruled out or can be ignored on grounds of implausibility. A couple of explanations are still viable. One is that, once a thermonuclear instability is triggered, the burning front, for some mysterious reason, is unable to propagate quickly on the surface of a black hole candidate (§5.3). This explanation, however, involves a considerable degree of special pleading. Burning fronts move quite rapidly on the surfaces of neutron stars and white dwarfs, so why should they have trouble on black hole candidates whose surface gravities lie in between? The other possibility is that black hole candidates are made of non-interacting dark matter so that there is no hard surface where the accreting gas can accumulate and undergo a thermonuclear instability ( $\$ 5.4)$. However, in this case, the gas would still accumulate at the center and form a baryonic ball with a surface. We have carried out preliminary calculations of the bursting behavior of such objects and our results suggest that bursts should be common. Detailed results are awaited.

Leaving aside the possibilities mentioned above, by far the most plausible explanation for the lack of bursts in black hole candidates is that the objects simply have no surfaces. They must then have event horizons. Though not yet strong enough to qualify as proof, this line of reasoning must surely be considered compelling evidence for the reality of the event horizon.

This work was supported in part by NASA grant NAG 5-10780 and NSF grant AST 0307433 . 


\section{REFERENCES}

Abramowicz, M. A., Kluzniak, W., \& Lasota, J.-P. 2002, A\&A, 396, L31

Alford, M., Rajagopal, K., \& Wilczek, F. 1998, Phys. Lett. B, 422, 247

Bahcall, S., Lynn, B. W., \& Selipsky, S. L. 1990, ApJ, 362, 251

Bildsten, L. 1998, in The Many Faces of Neutron Stars, ed. A. Alpar, L. Buccheri, \& J. van Paradijs (Dordrecht: Kluwer), 419

Bodmer, A. R. 1971, Phys. Rev. D, 4, 1601

Casares, J., Charles, P. A., \& Naylor, T. 1992, Nature, 355, 614

Cornelisse, R., in’t Zand, J. J. M., Verbunt, F., Kuulkers, E., Heise, J., et al. 2003, A\&A, 405, 1033

Done, C., \& Gierlinski, M. 2003, MNRAS, 342, 1041

Garcia, M. R., McClintock, J. E., Narayan, R., Callanan, P., Barret, D., \& Murray, S. S. 2001, ApJ, 553, L47

Gebhardt, K., Richstone, D., Tremaine, S., Lauer, T. R., Bender, R., Bower, G., Dressler, A., et al. 2003, ApJ, 583, 92

Ghez, A. M., Duchjne, G., Matthews, K., Hornstein, S. D., Tanner, A., Larkin, J., Morris, M., et al. 2003, ApJ, 586, L127

Glendenning, N. K. 2000, Compact Stars, Nuclear Physics, Particle Physics, and General Relativity, 2nd ed. (Berlin:Springer)

Grindlay, J., Gursky, H., Schnopper, H., Parsignault, D. R., Heise, J., Brinkman, A. C., \& Schrijver, J. 1976, ApJ, 205, L127

Hansen, C. J., \& van Horn, H. M. 1975, ApJ, 195, 735

Lewin, W. H. G., van Paradijs, J., \& Taam, R. E. 1993, Space Sci. Rev., 62, 223

McClintock, J. E., Narayan, R., Garcia, M. R., Orosz, J. A., Remillard, R. A., \& Murray, S. S. 2003, ApJ, 593, 435

McClintock, J. E., \& Remillard, R. A. 1986, ApJ, 308, 110

Narayan, R., Garcia, M. R., \& McClintock, J. E. 1997, ApJ, 478, L79 
Narayan, R., Garcia, M. R., \& McClintock, J. E. 2002, Proc. IX Marcel Grossmann Meeting: On Recent Developments in Theoretical and Experimental General Relativity, Gravitation and Relativistic Field Theories, eds V. Gurzadyan, R. Jantzen and R. Ruffini, World Scientific, Singapore, p405 (astro-ph/0107387)

Narayan, R., \& Heyl, J. S. 2002, ApJ, 574, L139

Narayan, R., \& Heyl, J. S. 2003, ApJ, 599, in press (astro-ph/0303447)

Rapp, R., Schafer, T., Shuryak, E., \& Velkovsky, M. 1998, Phys. Rev. Lett., 81, 43

Schödel, R., Ott, T., Genzel, R., Hofmann, R., Lehnert, M., Eckart, A., Mouawad, N., et al. 2002, Nature, 419, 694

Shapiro, S. L., \& Teukolsky, S. A. 1983, Black Holes, White Dwarfs, and Neutron Stars (New York: Wiley-Interscience)

Spitkovsky, A., Levin, Y., \& Ushomirsky, G. 2002, ApJ, 566, 1018

Sunyaev, R., \& Revnivtsev, M. 2000, A\&A, 358, 617

Tournear, D., Raffauf, E., Bloom, E. D., Focke, W., Giebels, B., Godfrey, G., Saz Parkinson, P. M., et al. 2003, ApJ, 595, 1058

van Paradijs, J., Penninx, W., \& Lewin, W. H. G. 1988, MNRAS, 233, 437

Witten, E. 1984, Phys. Rev. D, 30, 272 
Fig. 1.- Computer graphics image of an X-ray binary (courtesy: Robert Hynes, 2002). On the right is the donor secondary star from which mass is transferred via a narrow stream to the compact primary star on the left. The gas goes into orbit around the primary, and then spirals in via an accretion disk to accrete onto the compact star at the center. Some of the accreting gas is occasionally ejected in twin jets, as shown. 


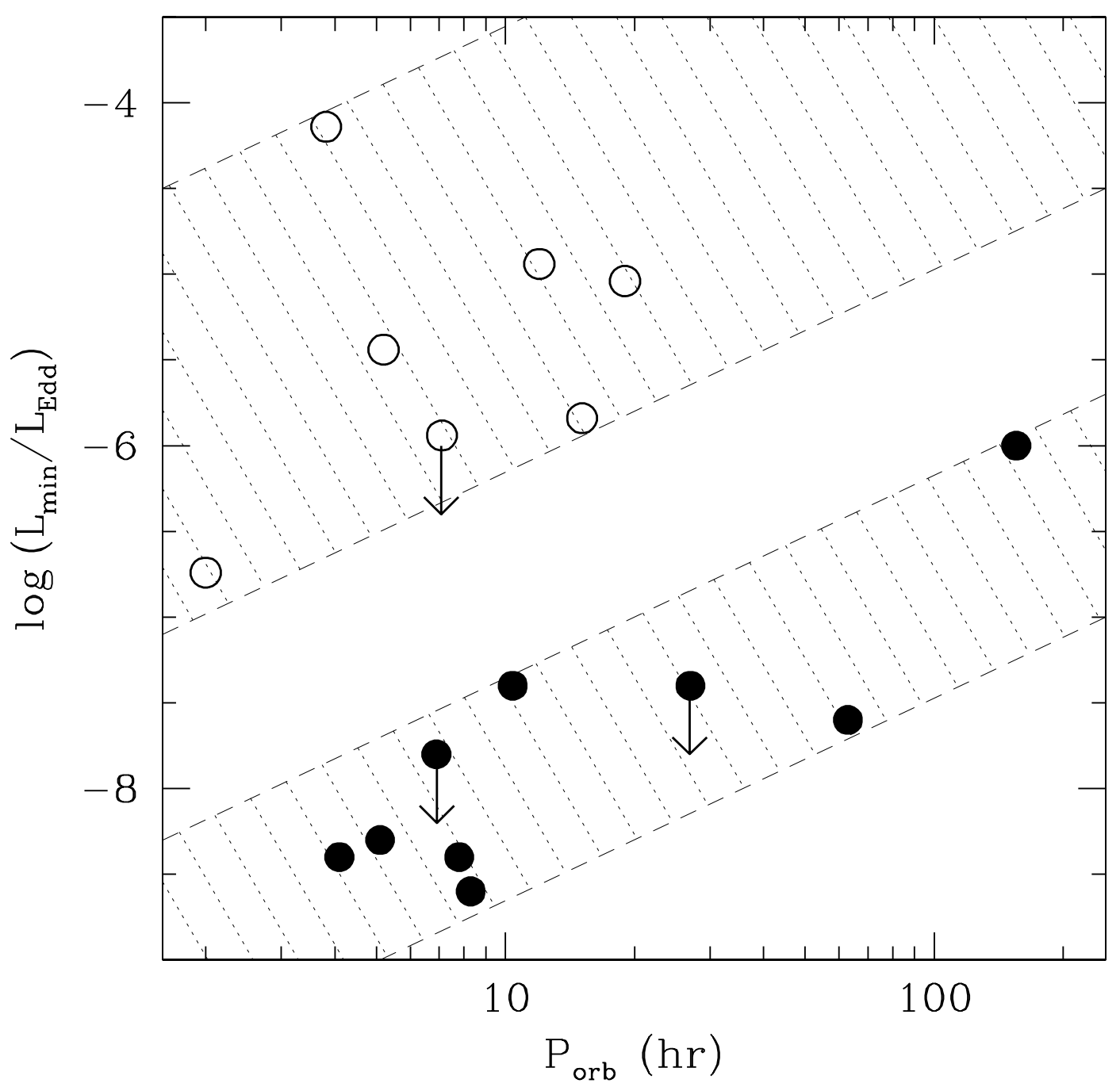

Fig. 2.- Luminosities in quiescence, measured in Eddington units, plotted against the orbital period, for 16 SXTs for which data are available (taken from McClintock et al. 2003). The open circles correspond to SXTs with neutron star primaries and the filled circles to systems with black hole candidates (identified by mass). The shaded bands have been drawn to emphasize that the two classes of systems are well-separated on the plot. Note that the vertical axis is in logarithmic units, so that quiescent black hole candidates are orders of magnitude dimmer than quiescent neutron stars. The large difference is arguably because black hole candidates have event horizons whereas neutron stars have surfaces (Narayan, Garcia \& McClintock 2002, and references therein). 


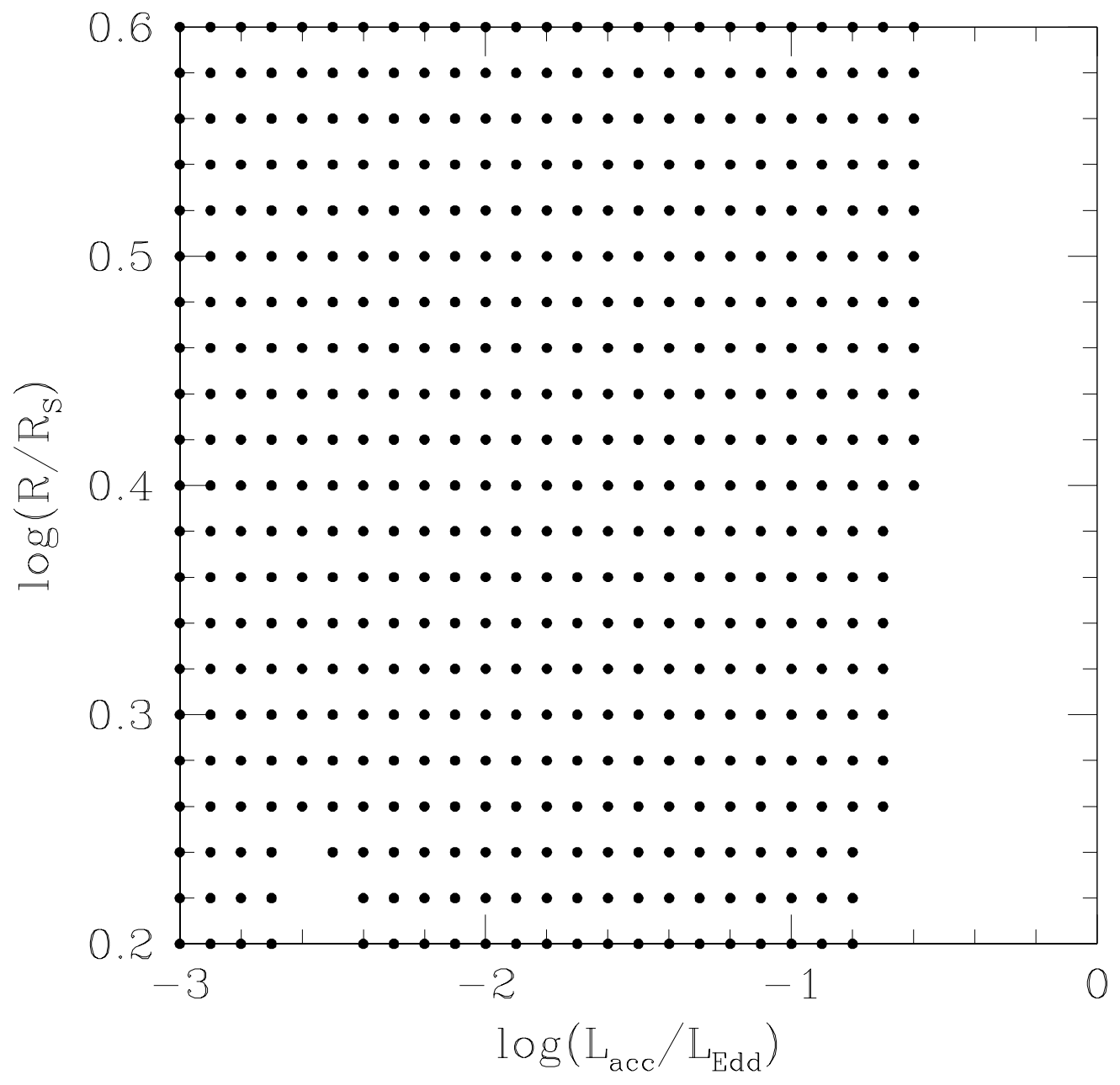

Fig. 3.- Results on the thermonuclear stability of accreting gas for a grid of neutron star models (Narayan \& Heyl 2003). The neutron star is assumed to have a mass of $1.4 M_{\odot}$ and a core temperature of $10^{8} \mathrm{~K}$. The models correspond to a range of stellar radii from $10^{0.2} R_{S}=6.5 \mathrm{~km}$ to $10^{0.6} R_{S}=16.4 \mathrm{~km}$, where $R_{S}$ is the Schwarzschild radius, and a range of mass accretion rates from 0.001 Eddington to 1 Eddington. The dots correspond to models in which the accreting gas is thermonuclearly unstable and produces Type I bursts. The empty regions correspond to models in which the gas accretes stably without bursts. 


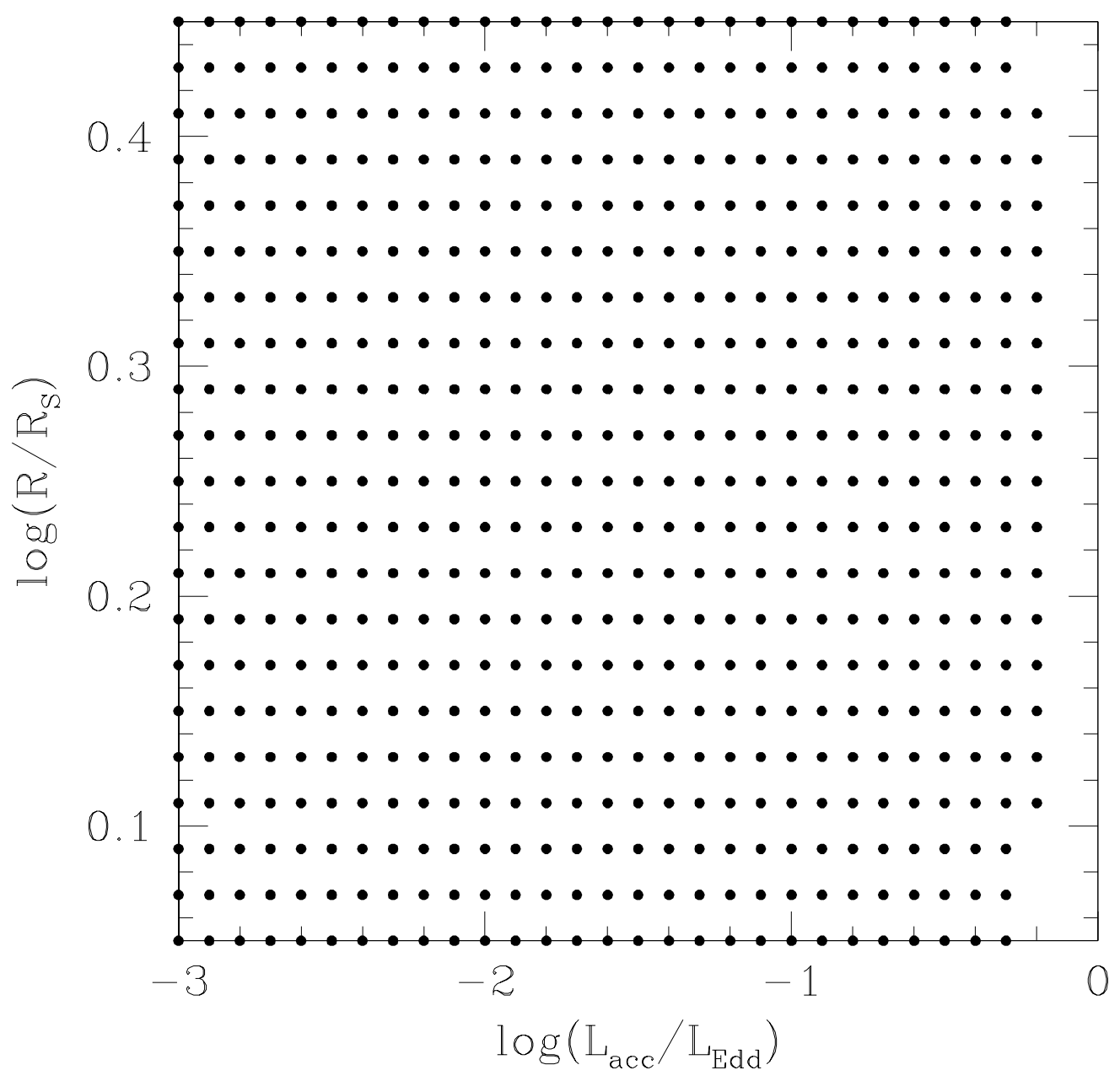

Fig. 4. - Similar to Fig. 3, but for a hypothetical black hole candidate with a surface. The star is assumed to have a mass of $10 M_{\odot}$ and a core temperature of $10^{7.5} \mathrm{~K}$. The models correspond to a range of radii from $(9 / 8) R_{S}=33 \mathrm{~km}$ to about $2.8 R_{S}=83 \mathrm{~km}$, and a range of accretion rates from 0.001 Eddington to 1 Eddington. The dots represent models in which this hypothetical object would produce Type I bursts, and the empty regions to models that are not expected to have bursts. 

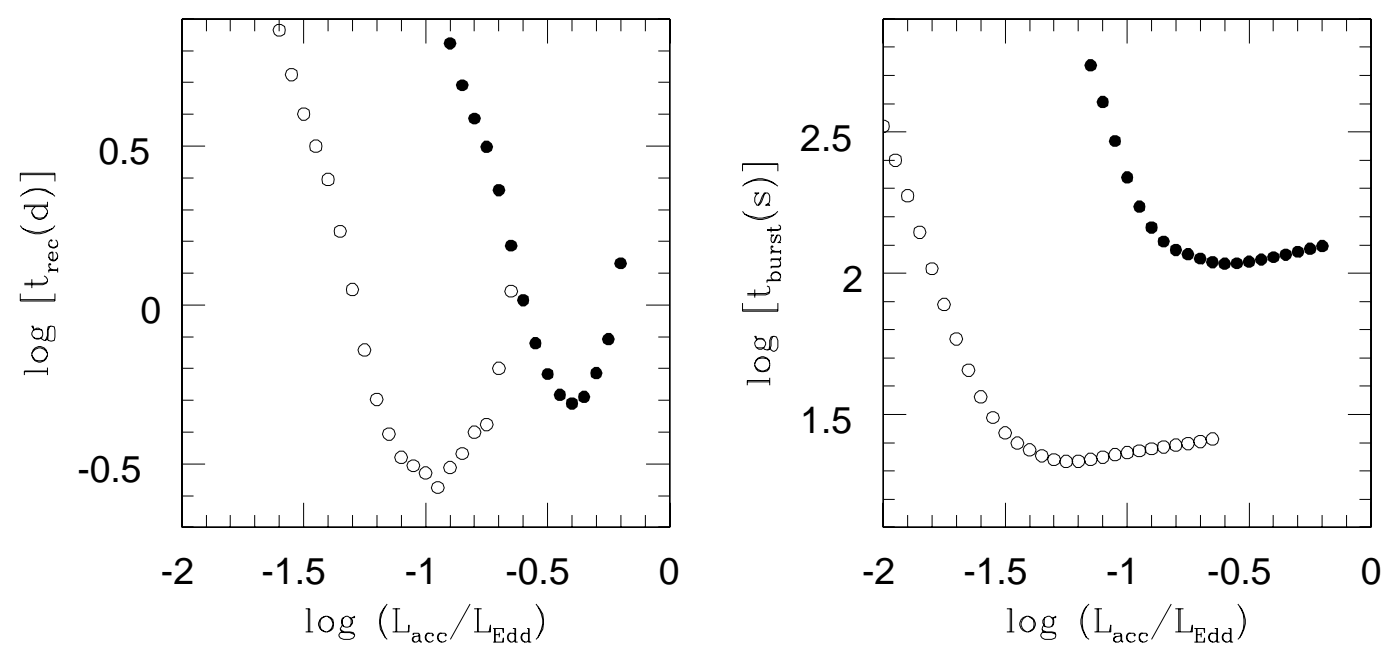

Fig. 5.- Shows on the left the recurrence time in days between bursts as a function of accretion rate for a $1.4 M_{\odot}$ neutron star with a radius of $10^{0.4} R_{S}=10.4 \mathrm{~km}$ (open circles) and a hypothetical $10 M_{\odot}$ black hole candidate with a surface at a radius of $2 R_{S}=59$ $\mathrm{km}$ (filled circles). Note that the black hole candidate is expected to produce bursts with reasonably short recurrence times for accretion rates $\sim 0.1-0.6$ Eddington. On the right are the corresponding burst durations in seconds, assuming that the burst energy comes out at the Eddington luminosity. 